\title{
Aprendizaje de la Educación Física Bilingüe a partir de las Metas de Logro y el Clima de Aprendizaje
}

\author{
Antonio BAENA-ExTREMERA \\ Universidad de Granada \\ MANuEl Gómez-LóPEZ \\ Universidad de Murcia \\ Antonio Granero-Gallegos \\ Universidad de Almería
}

Received: 17 November 2015/ Accepted: 14 February 2017

ISSN: 1697-7467

\begin{abstract}
RESUMEN: El objetivo de este trabajo fue hallar un modelo de predicción de la satisfacción/diversión hacia la Educación Física bilingüe, partiendo de las metas de logro del alumno y del clima motivacional. La nuestra fue de 328 estudiantes de secundaria (12-16 años). Se utilizó un cuestionario compuesto por varios instrumentos: Cuestionario del Clima Motivacional Percibido en el Deporte-2, Cuestionario de Metas de Logro 2x2 y Cuestionario de Satisfacción/Diversión con la Educación Física Bilingüe, analizándose Modelos de Ecuaciones Estructurales. La percepción del alumno de un clima motivacional de maestría predice la meta aproximación-maestría y ésta la satisfacción/diversión con las clases de Educación Física bilingüe.

Palabras clave: motivación, clima motivacional, metas de logro, diversión, bilingüismo.
\end{abstract}

\section{Learning Bilingual Physical Education from achievement goals and learning climate}

\begin{abstract}
The aim of this work was to find a model to predict satisfaction / fun to bilingual Physical Education, based on the student achievement goals and the motivational climate. Ours was 328 high school students (12-16 years). A questionnaire consisting of several instruments was used: Questionnaire Perceived Motivational Climate in Sport-2 Achievement Goals Questionnaire and Satisfaction Questionnaire 2x2 / Fun with Bilingual Physical Education, analyzing Structural Equation Modeling. The student's perception of a mastery motivational climate predicted mastery-approach goal and this satisfaction / fun classes Bilingual Physical Education
\end{abstract}

Keywords: motivation, motivational climate, achievement goals, fun, bilingualism.

\section{INTRODUCCIÓN}

En los últimos años las autoridades educativas han incentivado el bilingüismo en las aulas. Estos programas se han convertido en una de las prioridades para la adquisición de las competencias exigidas en los Reales Decretos de las Enseñanzas Mínimas (Cepero, García, \& López, 2013; Nikleva \& López-García, 2016). Cummins (2010) afirma que la educación 
bilingüe se refiere al uso de dos lenguas de instrucción en algún punto de la carrera escolar de un estudiante, utilizando cada lengua como medio de instrucción para enseñar el contenido de la asignatura, más que sólo el lenguaje en sí mismo. Señala, además, que el objetivo no es que el estudiante que adquiere la lengua logre el nivel de un hablante nativo, sino buscar un dominio de la segunda lengua que le permita utilizarla de manera adecuada en el contexto escolar.

La necesidad de una educación bilingüe queda notablemente reflejada en la legislación nacional (Ley Orgánica 8/2013 de la Mejora de la Calidad Educativa). Por ejemplo, el Real Decreto 126/2014, por el que se establece el currículum básico de la educación primaria, recoge que los gobiernos de la Unión Europea,

“... han programado diversas actuaciones comunitarias en materia de educación con el objetivo último de hacer posible que cada ciudadano posea un conocimiento práctico de al menos dos idiomas además de su lengua materna ..., en consonancia con esto, otorga a la capacitación para la comunicación en una o más lenguas extranjeras un lugar destacado entre los fines a cuya consecución está orientado nuestro actual sistema educativo".

Además, el RD citado recoge, para la etapa de primaria, una competencia específica en relación con el aprendizaje de lenguas extranjeras, denominada Competencia en Comunicación Lingüística (García, García, \& Yuste, 2012) y, por otro lado, hay que destacar el notable aumento de centros educativos que entran a formar parte de proyectos bilingües. Estos programas bilingües están basados en la integración de los contenidos y el aprendizaje de las lenguas extranjeras; los idiomas son utilizados como lenguas vehiculares mediante las que se adquieren los contenidos lingüísticos y académicos explicitados en el currículo. Estos programas han contribuido a la implantación de una nueva forma de enseñanza-aprendizaje, donde se requiere la participación activa del alumnado y un enfoque diferente en la forma de enseñar del profesorado (Freeman, Freeman, \& Mercuri, 2005). El bilingüismo fomenta la creatividad y flexibilidad cognitiva al obligar al alumnado a usar los recursos que poseen en dos idiomas (Lova, Bolarín, \& Porto, 2013). De hecho, existen numerosas investigaciones que han demostrado los beneficios académicos en cuanto al rendimiento escolar que obtienen los estudiantes bilingües en relación a los monolingües (Consejo Escolar de la Comunidad de Madrid, 2010; Thomas \& Collier, 2003); sin embargo, otros no encuentran resultados claramente positivos a favor del bilingüismo (Sotoca, 2014).

Teniendo en cuenta el artículo 15 del citado RD sobre autonomía pedagógica, el centro docente deberá organizar pedagógicamente qué asignaturas deben ser enseñadas a través de una lengua extranjera. Normalmente las asignaturas que se imparten en una segunda lengua varían entre Conocimiento del Medio, Educación Artística, Música y Educación Física (EF). Y es precisamente esta última, una de las más propicias para facilitar el aprendizaje del inglés como lengua extranjera a través del juego y del movimiento (Baena-Extremera \& Granero-Gallegos, 2015; Cepero et al., 2013; Ortiz, 2013). Debido a estas características y a la idiosincrasia particular de esta materia curricular en el aprendizaje de los discentes, la EF adquiere una importante función en el currículum (Coral, 2011). Esta idoneidad se debe, por un lado, al carácter altamente motivante, integrador, multicultural y social de los 
contenidos que la conforman (García et al., 2012) y, por otro, a su naturaleza interactiva, ya que permite la unión constante de lenguaje y acción, lo que ayuda a que el alumnado entienda gran parte de las instrucciones recibidas sin dominar todavía el inglés.

Aun así, el inglés como idioma instrumental puede transformarse involuntariamente en un elemento extraño, al no ser relevante para el estudiante, debido a que la lengua a aprender no forma parte de su vida diaria, afectando de este modo negativamente al interés por la asignatura y produciendo desmotivación (Liu \& Huang, 2011) y estrés (Hewitt \& Stefenson, 2011). Si a esta posibilidad se le une que la adolescencia es una etapa en la que se produce un descenso de la motivación (Franco, Pérez, \& Arrizabalaga, 2012) y de la implicación del alumnado en las clases de EF (Sallis \& McKenzie, 1991), es necesario encontrar planteamientos didácticos que fomenten la motivación y propicien experiencias positivas en el alumnado (Gómez-López, Granero-Gallegos, Baena-Extremera, \& Abraldes, 2014; Cuevas, García-Calvo, \& Contreras, 2013) ya que unos de los objetivos de la enseñanza es que a la vez que el discente aprende, se divierta y esté satisfecho con los conocimientos que adquiere.

Aunque el concepto de motivación es un término amplio y complejo, en el aprendizaje de segundas lenguas Brown (2000) asevera que la motivación es considerada como un factor no directamente relacionada con el lenguaje, pero que si afecta al proceso de adquisición del mismo. Diferentes estudios han demostrado que la motivación es uno de los factores clave que influyen en el éxito del aprendizaje de una segunda lengua (Ghazvini \& Khajehpour, 2011; Martínez, 2005). Por ejemplo, Ellis (1994) y Martínez (2005) afirman que el alumnado con un alto grado de motivación aprende antes y obtiene mejores resultados en el aprendizaje de una lengua extranjera. Del mismo modo, diferentes estudios han demostrado que cuando los estudiantes disfrutan y se divierten en las clases de EF, aumenta su motivación (Baena-Extremera, Granero-Gallegos, Bracho-Amador, \& Pérez-Quero, 2012; Gómez-López, Ruiz-Juan, García-Montes, Baena-Extremera, \& Granero-Gallegos, 2007).

Por otro lado, la satisfacción del alumnado con la escuela se relaciona con el rendimiento académico (Baños, Ortiz-Camacho, Baena-Extremera, \& Tristán-Rodríguez, 2017; Huebner \& Gilman, 2006), determinando el compromiso del estudiante con su trabajo escolar (Danielsen, Breivik, \& Wold, 2011). Incluso existe una estrecha relación entre la satisfacción y el compromiso del alumnado que decide aprender un nuevo idioma (Busse \& Walter, 2013). Así, se aprecia la importancia que tiene para el aprendizaje de un idioma (segunda lengua) el trabajo a través de otras materias, como la EF.

Para responder a este reto, la teoría de las Metas de Logro (Nicholls, 1984, 1989) constituye un marco útil para comprender la motivación y los comportamientos del alumnado. Esta teoría, es de sobra conocida por sus aplicaciones en la escuela y por la cantidad de investigaciones que la utilizan (Cañabate, Torralba, Cachón, \& Zagalaz, 2014). A grandes rasgos, esta teoría pretende descifrar la conducta en entornos de logro como las clases de $\mathrm{EF}$, analizando los distintos factores personales/disposicionales (orientación disposicional a la tarea o al ego) y ambientales/sociales (clima motivacional implicante al aprendizaje o al rendimiento generado por los agentes colindantes al alumno) que influyen en la motivación de logro del sujeto.

Con respecto a la orientación disposicional del alumnado, diferentes estudios han demostrado que el alumnado orientado a la tarea tiende a divertirse y a disfrutar con la práctica de actividades físico-deportivas en las clases de EF, al contrario de aquellos orientados al ego, que sienten aburrimiento (Duda \& Nicholls, 1992; Fernández-Río, Méndez-Giménez, Cecchini, \& González, 2012). 
En cuanto al clima motivacional percibido por el alumnado, subrayar que diferentes estudios han demostrado que un clima implicante al aprendizaje conlleva entre el alumnado altas dosis de diversión y motivación (Duda \& Nicholls, 1992; Papaioannou \& Theodorakis, 1996), al igual que el clima escolar influye en el rendimiento académico (Garrido, Jiménez, Landa, Páez, \& Ruiz, 2013; Giraldo \& Mera, 2000).

Por tanto, y una vez comprobada la importancia que tiene para el profesorado en general, y para el de EF en particular, el conocimiento, tanto las orientaciones de meta de su alumnado como del clima motivacional que afirman percibir en las clases, el objetivo del presente estudio fue hallar un modelo de predicción de la satisfacción y diversión hacia la EF bilingüe, partiendo de las metas de logro del alumnado y del clima motivacional creado por el docente. Se hipotetiza que la percepción de un clima de maestría predice la meta aproximación-maestría y ésta a su vez la satisfacción/diversión con las clases de EF en este idioma. Finalmente, hay que subrayar el reducido número de investigaciones sobre el aprendizaje de una segunda lengua a través de las clases de EF con estas variables.

\section{MÉtodo}

La muestra estuvo compuesta por un total de 328 estudiantes de entre 12 y 16 años $(M$ $=13.37 ; D T=1.40): 153$ chicos y 175 chicas, todos en centros públicos andaluces (Almería, Granada, Córdoba y Jaén) y en educación secundaria obligatoria en el momento de la investigación. Debido a la naturaleza de esta investigación, y de las posibilidades para acceder a la muestra, la selección de la misma fue de tipo no probabilístico y por conveniencia.

\subsection{Instrumentos}

Se utilizó Cuestionario del Clima Motivacional Percibido en el Deporte-2 (PMCSQ-2) adaptado al castellano por Cecchini, González, López, y Brustad (2005) del original de Newton, Duda, y Yin (2000), usando concretamente su versión adaptada a la EF de GonzálezCutre, Sicilia, y Moreno (2008). Este instrumento consta de 33 ítems para medir el clima motivacional de los estudiantes en clases de EF en dos dimensiones: percepción de un clima motivacional que implica al ego (16 ítems) y percepción de un clima motivacional que implica a la tarea (17 ítems). Los estudiantes deben responder según su grado de acuerdo o desacuerdo en una escala de cinco puntos tipo Likert que oscila entre (1) totalmente en desacuerdo y (5) тиy de acuerdo. Estudios anteriores han demostrado la validez interna de la estructura factorial del instrumento, así como su fiabilidad en el campo de EF (GonzálezCutre et al., 2008). En este estudio, la consistencia interna de la subescala de clima ego fue alfa de Cronbach $(\alpha)=.91$ y la de clima tarea, $\alpha=.89$. El análisis factorial confirmatorio (AFC) del instrumento mostró unos buenos indicadores: chi-cuadrado/grados de libertad ( $\mathrm{c}^{2} /$ $\mathrm{gl})=2.36$; índice de bondad de ajuste (GFI, por sus siglas en inglés $)=.96$; índice de ajuste normalizado (NFI, por sus siglas en inglés) $=.95$; índice de ajuste no normativo (NNFI, por sus siglas en inglés $)=.96$; índice de ajuste comparativo $(\mathrm{CFI})=.97$; error de aproximación cuadrático medio (RMSEA, por sus siglas en inglés) $=.05$. 
Cuestionario de Metas de Logro 2x2. Se utilizó la versión adaptada a la EF (AGQ) (Moreno, González-Cutre, \& Sicilia, 2008), de la versión de Wang, Biddle, y Elliot (2007), elaborada para medir orientaciones de metas de logro del alumnado de esta asignatura. Este cuestionario consta de un total de 12 ítems, compuesto de cuatro subescalas (tres ítems por factor): aproximación-maestría (AM), evitación-maestría (EM), aproximación-rendimiento (AR) y evitación-rendimiento (ER). Las respuestas se recogen en escala Likert desde 1 (totalmente en desacuerdo) hasta 7 (totalmente de acuerdo). La consistencia interna fue: AM, $\alpha=.77$; $\mathrm{EM}, \alpha=.70 ; \mathrm{AR}, \alpha=.86 ; \mathrm{ER}, \alpha=.76$. Siguiendo la estructura original de cuatro factores y doce indicadores, se realizó un AFC de la escala que mostró unos aceptables indicadores de ajuste: $\mathrm{c}^{2} / \mathrm{gl}=3.12 ; \mathrm{GFI}=.97 ; \mathrm{NFI}=.96 ; \mathrm{NNFI}=.97$; CFI $=.98 ; \mathrm{RMSEA}=.04$.

Satisfacción/Diversión con la Educación Física Bilingüe. Se utilizó el cuestionario de Satisfacción/Diversión con la EF Bilingüe (SSI-PE) de Baena-Extremera y Granero-Gallegos (2015), de la versión originaria del Sport Satisfaction Instrument (SSI) (Duda \& Nicholls, 1992). Este instrumento presenta dos factores: satisfacción/diversión con la EF bilingüe (5 ítems) y aburrimiento con la EF bilingüe (3 ítems). Las respuestas se recogen en una escala tipo Likert con un rango que oscila desde (1) totalmente en desacuerdo y (5) totalmente de acuerdo. La consistencia interna en la subescala de satisfacción/diversión fue $\alpha=.83$, y en la de aburrimiento, $\alpha=.71$. El AFC de la escala mostró índices de ajuste excelentes: $\mathrm{c}^{2} / g l$ $=1.56 ; \mathrm{GFI}=.95 ; \mathrm{NFI}=.98 ; \mathrm{NNFI}=.98 ; \mathrm{CFI}=.99 ; \mathrm{RMSEA}=.04$.

\subsection{Procedimiento}

En primer lugar, se contactó con los equipos directivos de los centros educativos, así como con el profesorado de Educación Física de dichos institutos de educación secundaria con el objeto de solicitar su colaboración en el estudio. Tras explicar los objetivos del estudio, se obtuvo permiso por parte de la dirección de los centros, así como colaboración por parte del profesorado. Teniendo en cuenta la condición de menores de edad de los participantes, se requirió autorización previa por parte de sus padres, madres o tutores legales. El cuestionario definitivo fue aplicado en el aula, sin la presencia del docente de EF del centro, y en presencia de uno de los investigadores que participaron en este trabajo, quien informó al alumnado acerca de la pretensión del estudio, así como del carácter voluntario de su participación y del tratamiento confidencial de la información recabada. La recogida de los cuestionarios se realiza de forma individual, según van finalizando de rellenarlos, pues de esta manera se pueden detectar errores y verificar que ninguno de los ítems de las escalas queda sin responder.

\subsection{Análisis estadístico}

Primero se realizaron análisis descriptivos, análisis de correlaciones bivariadas para comprobar el grado de relación entre las distintas variables, análisis de consistencia interna a través del alfa de Cronbach. También se calcularon los índices de asimetría y curtosis, siendo estos, en general, próximos a cero $\mathrm{y}<2$, lo que indica semejanza con la curva normal de forma univariada y permite utilizar técnicas factoriales en el AFC. Estos cálculos 
se realizaron con el programa estadístico SPSS 22.0. Antes de llevar a cabo los AFC y los modelos estructurales, se realizó el cálculo del coeficiente de Mardia-Based-Kappa para estimar la normalidad multivariante. Finalmente, se realizaron los AFC de cada instrumento y los modelos de regresión estructural con el programa LISREL 8.80.

\section{Resultados}

\subsection{Medias, desviaciones típicas y análisis de correlación}

En la tabla 1 se muestran los estadísticos descriptivos de las variables utilizadas así como su correlación. El Clima Tarea (CT) presenta una media más alta que el Clima Ego (CE). Igualmente, el alumnado presenta una mayor Aproximación a la Maestría (AM) que Evitación Rendimiento (ER) o Evitación Maestría (EM), siendo la media más baja la correspondiente a Aproximación al Rendimiento (AR). En relación al factor del SSI-EF, los estudiantes encuestados muestran una importante Satisfacción/Diversión (SAT/D). En cuanto a las correlaciones, destacan como negativas y significativas la mantenida entre CT y CE, entre AM y EM, y entre los factores del SSI-PE. Entre las positivas y significativas, destaca la correlación entre CT y AM y EM, así como entre CE y EM y entre el AM y la $\mathrm{SAT} / \mathrm{D}$, entre otras.

Tabla 1. Estadísticos descriptivos y correlaciones entre las subescalas.

\begin{tabular}{|c|c|c|c|c|c|c|c|c|c|c|c|c|}
\hline & $M$ & $D T$ & $Q 1$ & $Q 2$ & 1 & 2 & 3 & 4 & 5 & 6 & 7 & 8 \\
\hline 1. CT & 3.90 & .67 & .45 & -.40 & & $-48 * *$ & $.62 * *$ & $.61 * *$ & .26 & $.40 * *$ & $.53 * *$ & .20 \\
\hline 2. $\mathrm{CE}$ & 2.20 & .77 & -.31 & -.44 & & & $-.41 * *$ & $.59 * *$ & $.501^{* *}$ & .43 & $-.36 * *$ & $.35^{*}$ \\
\hline 3. AM & 5.10 & 1.41 & -.58 & -.23 & & & & $-.60 * *$ & $.36^{*}$ & $.45 * *$ & $.58 * *$ & -.05 \\
\hline 4. EM & 4.53 & 1.54 & -.38 & -.47 & & & & & $.55^{* *}$ & $.55^{* *}$ & .02 & .02 \\
\hline 5. AR & 4.29 & 1.66 & -.17 & -.77 & & & & & & $.43 * *$ & .01 & .03 \\
\hline 6. ER & 4.80 & 1.43 & -.33 & -.40 & & & & & & & .01 & .03 \\
\hline 7. SAT/D & 3.91 & 1.12 & -.90 & -.04 & & & & & & & & $-.46^{* *}$ \\
\hline 8. ABU & 2.16 & 1.27 & .77 & -.54 & & & & & & & & \\
\hline
\end{tabular}


**La correlación es significativa al nivel .01 (bilateral). $M=$ media; $D T=$ desviación típica; $Q 1$ = asimetría; $Q 2$ = curtosis; $\mathrm{CT}=$ clima tarea; $\mathrm{CE}=$ clima ego; $\mathrm{AM}=$ aproximación-maestría; $\mathrm{EM}=$ evitación maestría; $\mathrm{AR}=$ aproximación rendimiento; $\mathrm{ER}=$ evitación rendimiento; $\mathrm{SAT} / \mathrm{D}=$ satisfacción/diversión; $\mathrm{ABU}=$ aburrimiento.

\subsection{Modelo de Ecuaciones Estructurales}

El siguiente paso es analizar los modelos regresión estructural (SEM) con el objeto de hallar un modelo predictivo que se ajuste al objetivo de esta investigación.

En primer lugar se analizó la normalidad multivariante en cada instrumento con el test de normalidad multivariante relativa (RMK) de PRELIS. Los valores mostraron la falta de normalidad de los datos (PMCSQ-2, Mardia-Based-Kappa = .196; AGQ, Mardia-Based-Kappa $=.238$; SSI-PE, Mardia-Based-Kappa $=.335$ ), obligando a utilizar estimadores robustos, como el método de estimación weighted least squares (WLS) del programa LISREL 8.80 de Jöreskog y Sörbom (2003). Se calculó para ello una matriz de correlaciones policóricas y una matriz de covarianzas asintóticas. Seguidamente, se testearon diversos modelos estructurales hasta responder al objetivo de la investigación. Para ello, se tuvieron en cuenta diversos índices de ajuste: $\chi^{2} / g 1$, GFI, NFI, y CFI. Como índice incremental, se calculó el RMSEA. En el caso del $\chi^{2} / \mathrm{gl}$ son considerados aceptables valores de ajuste del modelo $<5.0$; en los índices: GFI, NFI, NNFI y CFI se consideran aceptables valores $\geq .90$; en el RMSEA se consideran aceptables valores iguales o menores que .07 (Hu \& Bentler, 1999). Finalmente, los parámetros estimados se consideran significativos cuando el valor asociado al valor $t$ es superior a $1.96(p<.05)$. Con el objeto de analizar las relaciones e interacciones existentes entre las variables pertenecientes al modelo que se plantea y que obtienen valores favorables para su análisis (CT, CE, AM, EM, AR, ER, SAT/D, ABU), se ha utilizado el Modelo de Ecuaciones Estructurales. Atendiendo a las recomendaciones de diversos autores (e.g., MacIntosh, 2007), se formularon y analizaron varios modelos. Por este último motivo, al realizar el SEM se observó que la dimensión CT solo predecía la maestría, y el CE solo el rendimiento, debido, entre otros motivos, a que se obtenían $t$-values que no cumplían los requerimientos necesarios.

Se tuvieron en cuenta además los Índices de Modificación propuestos por el programa (output) para mejorar el modelo, de ahí, que se obtuvieran predicciones desde la EM hacia la AM, y de la ER hacia la EM, dejando fuera el ABU y mejorando notablemente el modelo inicial. Finalmente, como resultado que mejor ajuste presentaba, según las variables estudiadas, se obtuvo el presentado en la Figura 1, que muestra seis variables latentes con un total de 39 variables observadas. Los resultados de ajuste del modelo resultaron adecuados: $\mathrm{c}^{2} / \mathrm{gl}=$ $2.95 ; p<.001 ; \mathrm{GFI}=.91 ; \mathrm{NFI}=.95 ; \mathrm{NNFI}=.96 ; \mathrm{CFI}=.96 ; \mathrm{RMSEA}=.04$.

Estos datos se ajustan a los parámetros establecidos, por lo que podemos aceptar como bueno el modelo propuesto (Hu \& Bentler, 1999). De igual modo, la contribución de cada uno de los factores a la predicción de otras variables se examinó a través de los pesos de regresión estandarizados. El $t$-value asociado a cada peso se tomó como una medida de la contribución, de modo que valores superiores a 1.96 se consideran como significativos. 


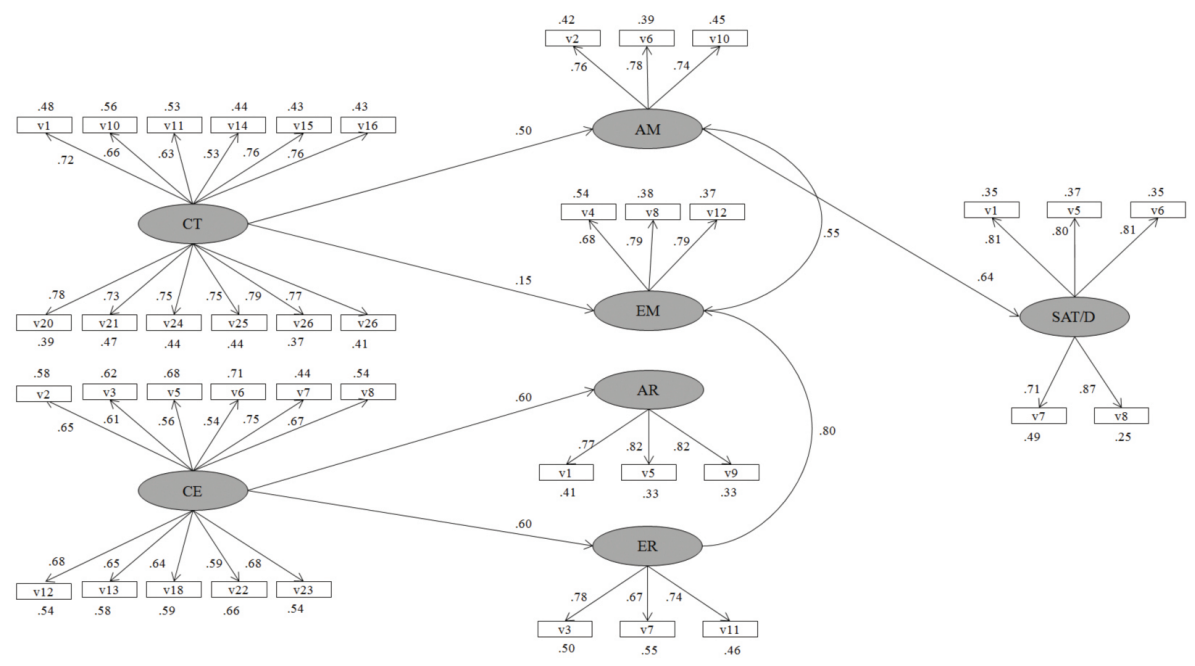

Figura 1: Modelo estructural compuesto por siete factores hipotetizados (CT = clima tarea; $C E=$ clima ego; $A M=$ aproximación-maestría; $E M=$ evitación maestría; $A R=$ aproximación rendimiento; $E R=$ evitación rendimiento; $S A T / D=$ satisfacción/diversión)

Se observa como el clima tarea predice la maestría, sobre todo en aproximación (.50), $\mathrm{y}$ menos en evitación (.15). Igualmente el clima ego predice el rendimiento, por igual, tanto en aproximación como en evitación (.60). Lo más destacable del modelo obtenido es que la satisfacción/diversión es predicha exclusivamente por la aproximación-maestría, que previamente es predicha por el clima tarea, o bien, como se observa, predicha previamente por la evitación-maestría (.55) y la evitación-rendimiento (ver Figura 1).

\section{Discusión y CONCLuSIONES}

El objetivo de esta investigación fue hallar un modelo de predicción a través de ecuaciones estructurales de la satisfacción/diversión de la EF Bilingüe.

Los resultados hallados demuestran que el alumnado posee una mayor aproximación-maestría y expresa una mayor satisfacción/diversión en las actividades que realizan en clase de EF bilingüe. En contra, los valores del aburrimiento son bajos, coincidiendo así con los estudios de Hernández, Velázquez, Martínez, Garoz, y López (2008) y Baena-Extremera et al. (2012), realizados con estudiantes monolingües. Estos resultados son positivos, puesto que el alumnado que disfruta en las clases de EF aumenta su compromiso con el aprendizaje de un nuevo idioma (Busse \& Walter, 2013) incrementando así su motivación (Baena et al., 2012; Gómez-López et al., 2007). Asimismo, como reflejan Ellis (1994) y Martínez (2005), el alumnado con un alto grado de motivación aprende antes y obtiene mejores resultados en el aprendizaje de una lengua extranjera. 
Respondiendo al objetivo principal de este trabajo, se ha demostrado que la percepción de un clima-maestría por parte del alumnado en sus clases de EF bilingüe, predice sobre todo la meta aproximación-maestría, la cual es predictora, a su vez, de la satisfacción/ diversión con las clases de EF bilingüe. De este modo se cumple la hipótesis planteada al inicio de este trabajo. Esta relación entre el clima motivacional y las orientaciones de meta fue expuesta anteriormente por Cury, Da Fonséca, Rufo, y Sarrazin (2002) y Wang, Liu, Chatzisarantis, y Lim (2010), en el ámbito educativo, aunque no en un contexto bilingüe, novedad que se aporta en esta investigación. Igualmente, Baena-Extremera y Granero-Gallegos (2015b) y Wang et al. (2010) hicieron hincapié en el efecto positivo que tiene la meta de aproximación-maestría en la diversión en EF, pero como en el caso anterior, aún no se disponen de datos sobre la EF bilingüe, vacío que este trabajo contribuye a subsanar. En este último estudio citado, se confirmó que la percepción de un clima de maestría predice la adopción de metas de aproximación-maestría y evitación-maestría, al tiempo que un clima de rendimiento se relaciona con las metas de aproximación-rendimiento y evitación-rendimiento. Asimismo, las metas de aproximación-maestría tienen fuertes efectos positivos en la diversión, mientras que las metas de evitación-rendimiento tienen pequeños efectos negativos. Por otro lado, Conroy, Kaye, y Coatsworth (2006) demostraron que mientras que las metas de aproximación-maestría se relacionan de forma positiva con un clima de maestría, y las metas de aproximación-rendimiento se relacionan de forma positiva con la percepción de un clima motivacional de rendimiento, las metas de evitación-rendimiento y evitación-maestría se relacionan con la desmotivación.

En este estudio también se demuestra que la meta aproximación-maestría puede ser predicha previamente por la evitación-maestría y la evitación-rendimiento, la cual a su vez es predicha por el clima-rendimiento. Estos resultados van en la línea de lo confirmado por Wang et al. (2007) y Méndez-Giménez, Fernández-Río, Cecchini, y González (2013), en el sentido de que la adopción de metas de evitación combinadas con la adopción de metas de aproximación se mostró como el perfil motivacional más adaptativo en el ámbito educativo. Por tanto, se sugiere que el docente de EF bilingüe fomente una combinación de orientaciones motivacionales, priorizando las metas de maestría, pero sin menospreciar la promoción asociada de metas de rendimiento (Méndez-Giménez et al., 2013) y, en este caso la de evitación, al objeto de provocar consecuencias motivacionales positivas.

En contra de los resultados expuestos, Cuevas et al. (2013) sugieren que las intervenciones docentes deben promover no solo las metas de maestría a través del clima motivacional, sino, también, en determinados momentos, la promoción de la aproximación-rendimiento, con el fin de crear patrones motivacionales adaptativos, caracterizados por una actitud positiva hacia la actividad, búsqueda de retos constantes, baja ansiedad y persistencia en el aprendizaje, características de interés para la enseñanza de los idiomas. Anteriormente otros autores también reflejaron que el alumnado que afirmaba divertirse más en las clases era el que percibía un clima motivacional de maestría (Duda \& Nicholls, 1992; Papaioannou \& Theodorakis, 1996).

Por todo ello, y a modo de conclusión, los resultados de este estudio indican que si el profesorado quiere potenciar en los estudiantes un mayor sentimiento de satisfacción/ diversión con las clases de EF bilingüe, es necesario una metodología de trabajo que propicie el paso de la enseñanza del inglés a la enseñanza en inglés (Lova et al., 2013). Una estrategia adecuada sería promover un clima motivacional de implicación en la tarea, que 
oriente al alumnado hacia la adopción de metas de maestría en las clases. De esta manera se reafirma el rol fundamental que desempeña el profesorado de EF bilingüe en la creación de un ambiente de clase que fomente el compromiso del estudiante hacia las actividades de clase y el aprendizaje de una segunda lengua.

Debido a las limitaciones del presente estudio, se propone la realización de futuras investigaciones en las que se amplíe la muestra para poder generalizar y comparar los resultados y se incluyan, además, otras variables relevantes en el ámbito académico relacionadas con la diversión, como la disciplina (Moreno, Zomeño, Marín, Ruiz, \& Cervelló, 2013) y el rendimiento o fracaso/éxito académico (Fernández, 2004). Asimismo, estudios futuros deberían plantear diseños de intervención con el fin de analizar la predicción de los climas motivacionales sobre las metas múltiples y la diversión/satisfacción en las clases de EF.

\section{REFERENCIAS}

Baena-Extremera, A. y Granero-Gallegos, A. (2015a). "Versión española del Sport Satisfaction Instrument (SSI) adaptado al aprendizaje de la Educación Física bilingüe en Inglés", en Porta Linguarum, 24: 63-76.

Baena-Extremera, A. y Granero-Gallegos, A. (2015b). "Modelo de predicción de la satisfacción con la Educación Física y la Escuela", en Revista de Psicodidáctica, 20(1): 177-192.

Baena-Extremera, A., Granero-Gallegos, A, Bracho-Amador, C., y Pérez-Quero, F. J. (2012). "Versión española del Sport Satisfaction Instrument (SSI) adaptado a la Educación Física", en Revista de Psicodidáctica, 17: 377-396.

Baños, R., Ortiz-Camacho, M. M., Baena-Extremera, A., y Tristán-Rodríguez, J. L. (2017). “Satisfacción, motivación y rendimiento académico en estudiantes de Secundaria y Bachillerato: antecedentes, diseño, metodología y propuesta de análisis para un trabajo de investigación”, en Espiral. Cuadernos del Profesorado, 10(20): 40-50.

Brown, H. D. (2000). Principles of language learning and teaching. New York: Addison Wesley-Longman.

Busse, V. y Walter, C. (2013). "Foreign Language Learning Motivation in Higher Education: A Longitudinal Study of Motivational Changes and Their Causes", en The Modern Language Journal, 97(2): 435-456.

Cañabate, D., Torralba, J. P., Cachón, J., Zagalaz, M. L. (2014). "Perfiles motivacionales en las sesiones de educación física, en Retos. Nuevas tendencias en educación física, deporte y recreación, 26: 34-39.

Cecchini, J. A., González, C., López Prado, J., y Brustad, R. J. (2005). "Relación del clima motivacional percibido con la orientación de meta, la motivación intrínseca y las opiniones y conductas de fair play", en Revista Mexicana de Psicología, 22: 469-479.

Cepero, M., García, A., y López, M. (2013). "Diseño de un programa de intervención bilingüe para el área de Educación Física basado en la competencia digital”, en Porta Linguarum, 19: $257-273$.

Conroy, D. E., Kaye, M. P., y Coatsworth, J. D. (2006). "Coaching climates and the destructive effects of mastery-avoidance achievement goals on situational motivation", en Journal of Sport and Exercise Psychology, 28: 69-92.

Consejo Escolar. Comunidad de Madrid. (2010). Los Programas de Enseñanza Bilingüe de la Comunidad de Madrid. Un estudio comparado. Recuperado de http:/www.madrid.org/edupubli. 
Coral, J. (2011). Mou-te i aprèn. Aprenentatge integrat de continguts d'educació física, salut i llengua anglesa. CLIL in Physical Education. En C. Escobar Urmeneta, AICLE-CLILEMILE Educació plurilingüe: Experiencias, research \& politiques. Barcelona: Universitat Autónoma de Barcelona. Servei de Publicacions, 181-192.

Cuevas, R., García-Calvo, T., y Contreras, O. (2013). "Perfiles motivacionales en Educación Física: una aproximación desde la teoría de las Metas de Logro 2x2", en Anales de Psicología, 29(3): 685-692.

Cummins, J. (2002). Lenguaje, poder y pedagogía: Niños y niñas bilingües entre dos fuegos. MEC: Morata.

Cummins, J. (2010). "Teaching for Transfer in Bilingual Education: Promoting Language Awareness and Literacy Engagement through Identity Texts". I Congreso Internacional sobre Bilingüismo en Centros Educativos. Madrid.

Cury, F., Da Fonséca, D., Rufo, M., y Sarrazin, P. (2002). "Perceptions of competence, implicit theory of ability, perception of motivational climate, and achievement goals: a test of the trichotomous conceptualization of endorsement of achievement motivation in the physical education setting", en Perceptual and Motor Skills, 95: 233-244.

Danielsen, A. G., Breivik, K. y Wold, B. (2011). "Do Perceived Academic Competence and School Satisfaction Mediate the Relationships Between Perceived Support Provided by Teachers and Classmates, and Academic Initiative?", en Scandinavian Journal of Educational Research, 55(4): 379-401.

Duda, J. L. y Nicholls, J. G. (1992). "Dimensions of achievement motivation in schoolwork and sport", en Journal of Educational Psychology, 84(3): 290-299.

Ellis, R. (1994). The study of second language acquisition. Oxford: Oxford University Press.

Fernández, M. (2004). "El clima motivacional de clase en estudiantes adolescentes de Buenos Aires”, en Revista Iberoamericana de Diagnóstico y Evaluación Psicológica, 18(2): 119-136.

Fernández-Río, F., Méndez-Giménez, A., Cecchini, J. A., y González, C. (2012). “Achievement Goals and Social Goals' Influence on Physical Education Students' Fair Play", en Revista de Psicodidáctica, 17: 73-91.

Franco, E., Pérez, J., y Arrizabalaga, A. (2012). "Motivación e intención de ser físicamente activos en jugadores de baloncesto en formación”. Cuadernos de Psicología del Deporte, 12(Supl. 1): 23-26.

Freeman, Y. S., Freeman, D. E., y Mercuri, S. P. (2005). Dual language essentials for teachers and administrators. Portsmouth, NH: Heinemann.

García, J. V., García, J. J., y Yuste, J. L. (2012). "Educación Física en inglés. Una propuesta para trabajar la higiene postural en Educación Primaria", en Retos. Nuevas tendencias en Educación Física, Deporte y Recreación, 22: 70-75.

Garrido, M., Jiménez, N., Landa, A., Páez, E. y Ruiz, M. (2013). "Factores que influyen en el rendimiento académico: la motivación como papel mediador en las estrategias de aprendizaje y clima escolar", en Revista electrónica de investigación y Docencia Creativa, 2: 17-25.

Ghazvini, S. D. y Khajehpour, M. (2011). "Attitudes and Motivation in learning English as Second Language in high school students", en Procedia-Social and Beavioral Sciences, 15: 1209-1213.

Giraldo, L. y Mera, R. (2000). "Clima social escolar: percepción del estudiante", en Revista Colombia Médica, 31: 23-27.

Gómez-López, M., Granero-Gallegos, A., Baena-Extremera, A., y Abraldes, J. A. (2014). “Análisis de los perfiles motivacionales y su relación con la importancia de la educación física en secundaria”, en Revista Iberoamericana de Diagnóstico y Evaluación Psicológica, 38: 9-27. 
Gómez-López, M., Ruiz-Juan, F., García, M. E., Baena, A., y Granero, A. (2007). “Opinión del alumnado universitario y de Educación Secundaria Postobligatoria sobre las clases de EF recibidas", en Retos. Nuevas tendencias en EF, Deporte y Recreación, 12: 58-61.

González-Cutre, D., Sicilia, A., y Moreno, J. A. (2008). "Modelo cognitivo-social de la motivación de logro en educación física", en Psicothema, 20: 642-651.

Guan, J., Xiang, P., McBride, R., y Bruene, A. (2006). "Achievement Goals, Social Goals and Students' Reported Persistence and Effort in High School Physical Education”, en Journal of Teaching in Physical Education, 25: 58-74

Hernández, J. L., Velázquez, R., Martínez, M. E., Garoz, I., y López, A. (2008). "Frecuencia de actividad física en niños y adolescentes: relación con su percepción de autoeficacia motriz, la práctica de su entorno social y su satisfacción con la Educación Física", en Infancia y Aprendizaje, 31: 79-92.

Hewitt, E. y Stephenson, J. (2011). "Foreign language anxiety and oral exam performance: A replication of Phillips's MLJ study", en The Modern Language Journal, 96: 170-189.

Hu, L. y Bentler, P. M. (1999). "Cutoff criteria for fit indexes in covariance structure analysis: Conventional criteria versus new alternatives”, en Structural Equation Modelling, 6: 1-55.

Huebner, E. S. y Gilman, R. (2006). "Students who like and dislike school", en Applied Research in Quality of Life, 2: 139-150.

Jöreskog, K. G. y Sörbom, D. (1993). Structural equation modeling with the SIMPLIS command language. Chicago: Scientific Software International.

Ley Orgánica 8/2013 de 9 de diciembre, de Mejora de la Calidad Educativa (BOE, nº 295 de 10 de diciembre)

Liu, M., y Huang, W. (2011). "An exploration of foreign language anxiety and English learning motivation", en Education Research International, 12: 1-8.

Lova, M., Bolarín, M. J., y Porto, M. (2013). "Programas bilingües en Educación Primaria: valoraciones de docentes", en Porta Linguarum, 20: 253-268.

MacIntosh, C. N. (2007). "Rethinking fit assessment in structural equation modelling: A commentary and elaboration on Barrett", en Personality and Individual Differences, 42: 859-867.

Martínez, M. (2005). “¿Qué relación guarda la motivación con los problemas de disciplina durante la adolescencia en los programas de educación bilingüe en EEUU?", en Porta Linguarum, 3: 21-34.

Méndez-Giménez, A., Fernández-Río, J., Cecchini, J. A., y González, C. (2013). "Perfiles motivacionales y sus consecuencias en educación física. Un estudio complementario de metas de logro 2x2 y autodeterminación", en Revista de Psicología del Deporte, 22: 29-38.

Moreno, J. A., González-Cutre, D., y Sicilia, A. (2008). "Metas de logro 2x2 en estudiantes españoles de Educación Física", en Revista de Educación, 347: 299-317.

Moreno, J. A., Zomeño, T. E., Marín, L. M., Ruiz, L. M., y Cervelló, E. (2013). "Percepción de la utilidad e importancia de la EF según la motivación generada por el docente", en Revista de Educación, 362: 380-401.

Newton, M., Duda, J. L., y Yin, Z. (2000). "Examination of the psychometric properties of the Perceived Motivational Climate in Sport Questionnaire-2 in a sample of female athletes", en Journal of Sport Sciences, 18: 275-290.

Nicholls, J. G. (1984). "Achievement motivation: Conceptions of ability, subjective experience, task choice, and performance", en Psychological Review, 91: 328-346.

Nicholls, J. G. (1989). The competitive ethos and democratic education. Cambridge, MASS: Harvard University Press.

Nikleva, D. G., y López-García, M. P. (2016). “Introducción a la enseñanza del español a in- 
migrantes: dimensiones didácticas e interculturales”, en Espiral. Cuadernos del Profesorado, 9(19): 31-43.

Ortiz, A. (2013). "Análisis contrastivo español-inglés de unidades fraseológicas usadas en la clase de Educación Física bilingüe", en Revista Funcae Digital, 33: 1-12.

Real Decreto 126/2014, de 28 de febrero, por el que se establece el currículo básico de Educación Primaria (BOE $\mathrm{n}^{\circ}$ 52, del 1 de marzo).

Papaioannou, A. y Theodorakis, Y. (1996). "A test of three models for the prediction of intention for participation in physical education lessons", en International Journal of Sport Psychology, 27: 383-399.

Sallis, J. F. y McKenzie, T. L. (1991). "Physical education's role in public health", en Research Quarterly for Exercise and Sport, 62: 124-137.

Sotoca, E. (2014). "La repercusión del bilingüismo en el rendimiento académico en alumnos de colegios públicos de la Comunidad de Madrid", en Revista Complutense de Educación, 25: 481-500.

Thomas, W. P. y Collier, V. P. (2003). “The Multiple Benefits of Dual Language”, en Educational Leadership, 61: 61-64.

Wang, C. K. J., Biddle, S. J. H., y Elliot A. J. (2007). "The 2x2 achievement goal framework in a Physical Education context", en Psychology of Sport and Exercise, 8: 147-168.

Wang, C. K. J., Liu, W. C., Chatzisarantis, N. L. D., y Lim, C. B. S. (2010). "Influence of perceived motivational climate on achievement goals in physical education: A structural equation mixture modeling analysis", en International Journal of Sport and Exercise Psychology, 32(3): 324-338. 